\title{
Spatial processing in a mental rotation task: Differences between high and low math-anxiety individuals
}

\author{
M. Isabel Núñez-Peña a, b, c, *, Belén González-Gómez ${ }^{\text {a, b }}$, Àngels Colomé b, d \\ ${ }^{a}$ Department of Social Psychology and Quantitative Psychology, Faculty of Psychology, University of Barcelona, Spain \\ ${ }^{\mathrm{b}}$ Institut de Neurociències, University of Barcelona, Spain \\ ${ }^{\mathrm{c}}$ Institut de Recerca Sant Joan de Déu, Spain \\ ${ }^{\mathrm{d}}$ Department of Cognition, Development and Educational Psychology, Faculty of Psychology, University of Barcelona, Spain
}

\section{A R T ICLE INFO}

\section{Keywords:}

Math anxiety

Spatial processing

Attentional control theory

ERPs

Rotation-related negativity

P3b

\begin{abstract}
A B S T R A C T
Previous studies suggested that highly math-anxious (HMA) individuals invest more attentional resources than their low math-anxious (LMA) peers in numerical tasks, and have worse spatial skills. We aimed to explore whether they also need to apply more resources in spatial tasks. In this study, HMA and LMA individuals saw normal or mirror-reversed letters in six orientations and made mirror-normal decisions. In both groups, response times and errors increased with angular deviation from upright and the ERP mental rotation effect was found. However, HMAs were slower to respond than their LMA counterparts. Interestingly, the HMA group showed a larger P3b in greater deviations for normal letters and in all mirrored letters. Since P3b amplitude reflects the attentional resources invested in the categorization of relevant stimuli, HMA individuals may need to devote more processing effort than their LMA peers when performing mental rotation. This finding is consistent with the Attentional Control Theory.
\end{abstract}

\section{Introduction}

Mathematical skills are essential in modern technological societies but, unfortunately, many people find it difficult to learn and use mathematics. Among them, a large number suffer from math anxiety: that is, they feel tension, apprehension or fear when faced with tasks involving numbers and math use (Richardson \& Suinn, 1972; Suárez-Pellicioni, Núñez-Peña, \& Colomé, 2016). These feelings are present in highly math-anxious individuals not only in academic contexts involving mathematical problem-solving but also in everyday and professional settings (Ashcraft \& Ridley, 2005).

While most of the existing body of evidence on math-anxious individuals has focused on their math performance, a number of studies have examined the relationship between math anxiety and spatial ability (e.g., Ferguson, Maloney, Fugelsang, \& Risko, 2015; Maloney, Waechter, Risko, \& Fugelsang, 2012; Sokolowski, Hawes, \& Lyons, 2019). Spatial ability is defined as "the ability to generate, retain, retrieve and transform well-structured visual images" (Lohman, 1994, p. 1000). The relation between math abilities and spatial skills is evident in both behavioral (for a review, see de Hevia, Vallar, \& Girelli, 2008) and neuropsychological measures (e.g., Hubbard, Piazza, Pinel, \& Dehaene, 2005; Pinel, Piazza, Le Bihan, \& Dehaene, 2004). People with high spatial ability perform better on mathematical tests (Mix \& Cheng, 2012), and important theories such as the triple-code model claim that participants rely on visuo-spatial processes when they are engaged in arithmetic and numerical processing (e.g., Dehaene, 1992). Moreover, tasks that require either number processing or spatial transformations tend to activate structures within the parietal lobes (Hubbard et al., 2005).

Regarding the relationship between math anxiety and spatial ability, Maloney et al. (2012) suggested that the higher math anxiety in females may in part be mediated by gender differences in visuo-spatial processing ability. Furthermore, Ferguson et al. (2015) and Maloney et al. (2012) demonstrated that higher math anxiety is associated with worse self-reported spatial processing ability, and poorer performance in spatial processing tasks. This spatial deficit in highly math-anxious individuals was specific to small-scale spatial skills (e.g., mental rotation) and to the shared aspects of small- and large-scale spatial abil-

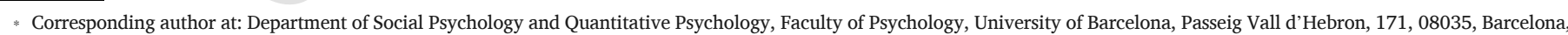
Spain.

Email address: inunez@ub.edu (M.Isabel Núñez-Peña)
} 
ities. ${ }^{1}$ Moreover, Ferguson et al. (2015) found a positive correlation between math anxiety and spatial anxiety (i.e., anxiety about environmental navigation), although interestingly the relation between math anxiety and performance in their small-scale spatial task was maintained even after controlling for spatial anxiety.

Recent studies have suggested that highly math-anxious individuals might have an attentional-control deficit that would make them less efficient in numerical tasks than their low math-anxious peers (Suárez-Pellicioni, Núñez-Peña, \& Colomé, 2014; Suárez-Pellicioni, Núñez-Peña, \& Colomé, 2013; Suárez-Pellicioni, Núñez-Peña, \& Colomé, 2015). Specifically, Suárez-Pellicioni and colleagues proposed that this deficit would force individuals high in math anxiety to invest more attentional resources in math-related tasks. Suárez-Pellicioni et al.'s proposal is based on the Attentional Control Theory (ACT; Eysenck, Derakshan, Santos, \& Calvo, 2007), which was developed from Processing Efficiency Theory (PET; Eysenck \& Calvo, 1992). According to the ACT, anxiety affects performance because it impairs the attentional control function of the central executive system. Highly anxious individuals allocate their attentional resources to threat-relevant stimuli that may be internal (e.g., worries) or external (e.g., task-irrelevant distractors), so they need to invest more resources in order to solve the task as accurately as their low-anxiety peers. ACT and PET distinguish between performance effectiveness and processing efficiency. The former refers to the result of the performance (i.e., how well a person performs a task; usually measured by means of accuracy) whereas the latter refers to the amount of effort or cognitive resources invested in order to perform the task (usually measured by means of response time). According to these theories, anxiety would impair processing efficiency more than performance effectiveness, in so far as when a highly-anxious person faces an anxious task, he or she would have to invest more processing resources to perform it as accurately as a person with low anxiety.

in the present study, we wanted to examine whether high math-anxiety (A) individuals need to invest more attentional resources than their low math-anxiety (LMA) peers to perform small-scale spatial tasks. This attentional control deficit could be one of the factors contributing to HMA individuals' difficulties in performing mental rotation tasks (Ferguson et al., 2015; Maloney et al., 2012). In the present experiment, we studied this issue by examining spatial processing ability in HMA individuals in a mental rotation task by means of event-related brain potential (ERP) recording. This study presents two main novelties with respect to previous investigations in this field. First, we used a computerized version of the mental rotation task that allowed us to measure the mental rotation effect both on accuracy and on response time. In order to measure spatial processing ability, previous studies used either paper-and-pencil self-report questionnaires (Ferguson et al., 2015; Maloney et al., 2012) or a computerized version of a mental rotation task where only accuracy was measured (Ferguson et al., 2015). Some studies have reported that HMA and LMA individuals differ more in processing efficiency than in effectiveness or, in other words, that HMA are slower than their LMA peers when performing math-related tasks but do not differ in accuracy (e.g., Núñez-Peña \& Suárez-Pellicioni, 2014; Suárez-Pellicioni et al., 2014). Therefore, it is relevant to measure response time in math anxiety studies. The second novelty of the present study is that event-related brain potentials (ERPs) were recorded. In contrast with the standard measures used in previous studies, the ERP technique allowed us to

1 Spatial ability is composed of two components (Hegarty et al., 2006; Wang, Cohen, \& Carr, 2014): (1) small-scale spatial abilities that are used for mental transformation of small shapes or manipulable objects (e.g., mental rotation) and (2) large-scale spatial abilities that are used to learn the layout of new environments and to physically navigate through space. study differences in brain response between high and low math-anxious individuals and provided us with a continuous measure of processing from stimulus onset to response.

Mental rotation is defined as the ability to imagine the rotation of an object without physically performing it. It has been linked to performance in several mathematical tasks, including mental arithmetic and geometry (Mix \& Cheng, 2012), and it is a strong predictor of later mathematics performance and success in the STEM (Science, Technology, Engineering and Mathematics) fields (Lubinski, 2010; Wai, Lubinski, \& Benbow, 2009). The mental rotation process is reflected both in response time (RT) and psychophysiological activity (i.e., ERPs). In a classical mental rotation task, visual stimuli (e.g., letters) in their normal or mirror-reversed version are presented rotated at different positions increasing in deviation from the upright, and participants are asked to indicate in which version the stimuli have been presented. RT increases with the required angle of rotation, an effect (RT mental rotation effect) first described by Shepard and Metzler (1971). It has been suggested that this increase in RT occurs because, in order to perform the task properly, the stimulus needs to be mentally rotated to place it in the upright position. In fact, this is what participants report when they are asked how they performed the task (Cooper \& Shepard, 1973). The mental rotation process also has an electrophysiological signature in the ERPs. As the angular disparity from upright increases, the amplitude becomes more negative over the parietal cortex in the interval ranging between 400 and 800 ms post-stimulus (Peronnet \& Farah, 1989). This effect has been replicated across a wide range of mental rotation tasks with different types of visual stimuli (Heil \& Rolke, 2002; Núñez-Peña, Aznar-Casanova, Linares, Corral, \& Escera, 2005; Thayer \& Johnson, 2006). It has been suggested that this slow negative component (the rotation-related negativity - RRN) is a psychophysiological correlate of the mental rotation process (Heil, 2002; Wijers, Otten, Feenstra, Mulder, \& Mulder, 1989) and it is superimposed on a strong late positive complex (P3b). Thus, the superposition of the two ERPs results in a positive wave. The P3b is not dependent on the rotation of the stimulus but it changes according to the presentation of a task-relevant stimulus (Rösler, Schumacher, \& Sojka, 1990; Wijers et al., 1989). P3b amplitude is proportional to the amount of attentional resources engaged in categorizing a given stimulus (Gray, Ambady, Lowenthal, \& Deldin, 2004; Johnson, 1988) and is strongly reduced when attention is directed away from the task (Duncan-Johnson \& Donchin, 1977; Johnson, 1988). Larger P3b amplitudes have been found in tasks requiring attentional control (e.g., task switching; Barceló, Periáñez, \& Nyhus, 2008). Therefore, $\mathrm{P} 3 \mathrm{~b}$ amplitude is considered an index of the cognitive effort applied during task processing, with larger amplitudes indicating more attentional resources devoted to the task (Kok, 1997, 2001; Polich, 2007).

To examine whether HMA individuals need to invest more attentional resources than their LMA peers in spatial tasks, we recorded ERPs and behavioral measures while HMA and LMA individuals performed a mental rotation task. Participants had to indicate whether stimuli presented at different orientations $(0,50,100,150,210,260$ and 310 degrees) were normal or mirror-reversed letters. Our predictions were as follows: (1) we expected to replicate the mental rotation effect both behaviorally and in the brain response in both groups; (2) if, as proposed by the ACT, anxiety affects processing efficiency more than processing effectiveness, we expected the HMA group to be slower that their LMA peers (worse processing efficiency), but to show no significant group differences in hit rate (similar group processing effectiveness); and (3) because an increase in the P3b amplitude is considered a signature of the greater use of attentional resources, if the HMA group needs to devote more cognitive effort to perform the spatial task than their LMA counterparts, then we would expect a larger P3b for the HMA group. 
In the present study, three relevant variables were controlled: trait anxiety, spatial anxiety, and gender. Trait anxiety is positively related to math anxiety (Hembree, 1990) and according to the ACT (Eysenck et al., 2007) it affects attentional control. Moreover, spatial anxiety is positively related to math anxiety and negatively related to performance in spatial tasks (Lawton, 1994). Hence, if a math anxiety effect was found, it was important to ensure that it was not due to any of these variables. Regarding gender, differences between women and men have been described in spatial tasks in both behavioral and psychophysiological measures. Many studies have shown that males outperform females on spatial ability, especially in mental rotation tasks (Levine, Foley, Lourenco, Ehrlich, \& Ratliff, 2016; Lippa, Collaer, \& Peters, 2010; Reilly \& Neumann, 2013; Voyer, Voyer, \& Bryden, 1995). Regarding psychophysiological measures, gender differences in mental rotation tasks have also been reported (Gootjes, Bruggeling, Magnée, \& Van Strien, 2008; Mikhailova, Slavutskaya, \& Gerasimenko, 2012). For example, Gootjes et al. (2008) found that women were about $100 \mathrm{~ms}$ slower than men in a mental rotation task and that their ERP mental rotation effect (the rotation-related negativity) was delayed: it appeared in the $400-500 \mathrm{~ms}$ window for men, and in the 500-600 ms window for women. Therefore, to homogenize our sample only women were selected as participants in the present study. We chose women instead of men because they consistently report higher levels of math anxiety (Hembree, 1990).

\section{Methods}

\subsection{Participants}

Forty healthy female volunteers participated in this study, half of them with a high level of math anxiety and the other half with a low level. They were selected from a sample of 770 students from the University of Barcelona who had previously been assessed for trait anxiety and math anxiety in the framework of a larger project. One participant in the LMA group was excluded from the analysis due to technical problems during recording and two from the HMA group because they had an insufficient number of epochs in one of their ERP averages. Thus, the final sample included 19 LMA and 18 HMA participants.

The LMA group scored below the first quartile on the Shortened Mathematics Anxiety Rating Scale $(\mathrm{Q} 1=52$; mean $=45.10, \mathrm{SEM}=1.10)$, and their HMA peers above the third quartile $(\mathrm{Q} 3=77$; mean $=85.39$, SEM $=1.63)$. They were paired according to their scores on trait anxiety, so that they did not differ in this variable $(t(35)=1.38, p=.177)$, only in math anxiety $(t(35)=20.63, p<.001)$. Given that both kinds of anxiety are correlated (Hembree, 1990), we thus ensured that group differences in our results were not due to trait anxiety. Groups did not differ in age $(t(35)=1.49, p=.145)$ or handedness $\left(\chi^{2}(1)=.002, p=\right.$ .97). However, the HMA group showed more spatial anxiety than their LMA peers $(t(35)=2.99, p=.005)$. More detailed information about the two groups is provided in Table 1 .

All the participants had normal or corrected-to-normal visual acuity and none were taking any medication or substance that might affect

Table 1

Means and SEM (in brackets) for math anxiety, trait anxiety, age and spatial anxiety for the LMA and HMA groups. Number of right-handed individuals is also given.

\begin{tabular}{llllll}
\hline & $\begin{array}{l}\text { Math } \\
\text { anxiety }\end{array}$ & $\begin{array}{l}\text { Trait } \\
\text { anxiety }\end{array}$ & Age & $\begin{array}{l}\text { Spatial } \\
\text { anxiety }\end{array}$ & Handedness \\
\hline LMA & 45.10 & 21.26 & $\begin{array}{l}21.63 \\
(.31)\end{array}$ & $16.00(.94)$ & 18 \\
& $(1.10)$ & $(2.11)$ & 22.78 & $20.28(1.08)$ & 18 \\
HMA & 85.39 & 25.67 & $\begin{array}{l}22.78 \\
(.72)\end{array}$ & & \\
\hline
\end{tabular}

the electrophysiological response. Participants were not informed of the purpose of the study until they had finished the task. They signed an informed consent statement prior to the experiment and were paid for their participation. The complete experimental protocol was approved by the Ethics Committee of the University of Barcelona.

\subsection{Material}

\subsubsection{Shortened Mathematics Anxiety Rating Scale (sMARS) (Alexander} \& Martray, 1989)

The sMARS is a 25-item version of the Math Anxiety Rating Scale (MARS; Richardson \& Suinn, 1972). It measures anxiety by making participants imagine 25 situations that might cause math anxiety (e.g., "Thinking about the math exam I will have next week") and evaluate their level of anxiety in response to each of them. A five-point Likert scale, ranging from 1 (no anxiety) to 5 (high anxiety), is provided. The sum of all the items' scores proffers the total participant's score, which ranges from 25 to 125 points. In this study, we used the Spanish version of the sMARS (Núñez-Peña, Suárez-Pellicioni, Guilera, \& Mercadé-Carranza, 2013), which is known to have good psychometric properties (Cronbach's alpha was .94 and the intra-class correlation coefficient for 7-week test-retest reliability was .72).

\subsubsection{State-Trait Anxiety Inventory (STAI) (Spielberger, Gorsuch,} Lushene, Vagg, \& Jacobs, 1983)

The STAI is a scale that comprises 40 items, half of them used to measure state anxiety (STAI-S) and the other half to measure trait anxiety (STAI-T). Only the latter subscale, which measures a more stable tendency to respond with anxiety, was used in this study. The 20 statements in this sub-scale describe different emotions, and for each item respondents use a four-point Likert scale, ranging from 0 (almost never) to 3 (almost always), to indicate how they feel "in general". In this study, we used the Spanish version of the STAI-T (Spielberger, Gorsuch, \& Lushene, 2008), which is known to have good psychometric properties (Cronbach's alpha $=0.95$ and 20-day test-retest reliability with college students $=0.86$ ).

\subsubsection{Spatial Anxiety Scale (SAS) (Lawton, 1994)}

This is an eight-item scale that measures spatial anxiety. Participants must indicate on a five-point Likert scale how much anxiety they would feel in eight situations that require large-scale spatial ability (e.g., "Finding your way out of a complex arrangement of offices that you have visited for the first time"). The participant's score is the sum of the answers to the items (range 8-40), 40 being the maximum level of spatial anxiety. Although it assesses anxiety in situations requiring environmental navigation, we decided to use this scale because Ferguson et al. (2015) used it in a study similar to ours. Even if these authors found that only small-scale (and not large-scale) skills predicted math anxiety, they still found a significant correlation between SAS scores and mental rotation performance or math anxiety. Since there is no Spanish version of the SAS, we used the back-translation method to obtain the items. First, the original items were translated from English to Spanish (Spanish version), then a native English speaker translated the Spanish version into English and, finally, another native English speaker compared the two versions in English (original and translated) to verify that the items of the two versions conveyed the same ideas. In case of disagreement between versions, the English items were compared with the Spanish version and the conflict was resolved by consensus between translators.

\subsubsection{Mental rotation task}

A visual computerized task was used to evaluate the mental rotation process. This involved the presentation of four different characters (the uppercase letters F, G, P, and R) in their normal or mirrored version in 
different orientations $\left(0^{\circ}, 50^{\circ}, 100^{\circ}, 150^{\circ}, 210^{\circ}, 260^{\circ}\right.$ and $\left.310^{\circ}\right)$. Fig. 1 shows some examples of the stimuli used in the experiment.

Stimuli were shown in black Arial font on a white background. In the upright position, the letters had a height of $5.8 \mathrm{~cm}$ and a maximum width of $4.8 \mathrm{~cm}$, subtending a vertical visual angle of $2.2^{\circ}$ and a horizontal visual angle of $1.8^{\circ}$. The E-Prime 2.0 software (Psychology Software Tools Inc., Sharpsburg, PA) was used to control the presentation and timing of the stimuli, as well as to record the behavioral measurements (RT and accuracy).

\subsection{Procedure}

Participants were tested individually. Before preparing the electroencephalogram (EEG) recording, they signed the informed consent document and were asked about their handedness and age. EEG sensor electrodes were then attached and they were given the task instructions.

Participants were seated $150 \mathrm{~cm}$ away from the computer screen in an electrically shielded, sound-attenuating recording chamber and were asked to indicate whether the stimulus was presented in its normal or mirrored version by pressing one of the two buttons of the mouse. Response buttons were counterbalanced between subjects. The experimental session began with a training period of eight trials, where all the letters were presented, both in their normal and mirror-reversed version, so that the subjects were able to familiarize themselves with their appearance. They received feedback on their performance during training.

Each trial began with a fixation sign (a hash mark) shown for $500 \mathrm{~ms}$. Participants were previously asked to try to synchronize their blinks with the hash mark. Next, after a pause of $100 \mathrm{~ms}$, the test character appeared on the screen until the participant answered, or for a maximum of four seconds. The next trial began after $500 \mathrm{~ms}$. The recording session consisted of six blocks with 64 trials each (a total of 384 trials) and pauses of $30 \mathrm{~s}$ between them, in order to let participants rest and blink as much as they needed. All possible stimuli (four letters in two versions and four orientations) were randomly presented to each participant within each block. For orientations that were not upright, both clockwise and counterclockwise senses $\left(50^{\circ} / 310^{\circ}\right.$, $100^{\circ} / 260^{\circ}, 150^{\circ} / 210^{\circ}$ ) were presented once and eventually collapsed, while the upright stimuli $\left(0^{\circ}\right)$ were repeated twice in each block.

\subsection{Electrophysiological recording}

The EEG signal was recorded using the Scan 4.5 hardware and software (Compumedics Neuroscan, Inc., Herndon, VA), with 32 tin electrodes mounted in an elastic electro-cap. Electrodes were positioned according to the 10/10 International System: eight electrodes were placed over the midline sagittal plane sites at $\mathrm{Fpz}, \mathrm{Fz}, \mathrm{FCz}, \mathrm{Cz}, \mathrm{CPz}$, $\mathrm{Pz}, \mathrm{POz}$ and $\mathrm{Oz}$ locations, along with 12 lateral pairs of electrodes over standard sites in the prefrontal (FP1/FP2), frontal (F3/F4, F7/F8),

\section{$F \wedge \boldsymbol{* \cdots <}$

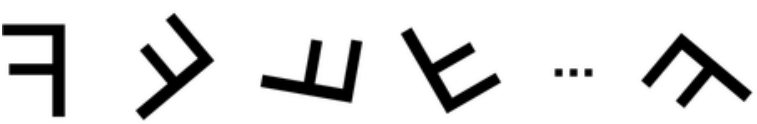 \\ $\begin{array}{llllll}0^{\circ} & 50^{\circ} & 100^{\circ} & 150^{\circ} & \cdots & 310^{\circ}\end{array}$}

Fig. 1. Normal and mirrored versions of the "F" letter in each orientation. fronto-central (FC3/FC4), frontotemporal (FT7/FT8), central (C3/C4), temporal (T7/T8), centro-parietal (CP3/CP4), temporo-parietal (TP7/ TP8), parietal (P3/P4, P7/P8) and occipital (O1/O2) positions. Likewise, the electro-cap was placed following the 10/10 International System, with $\mathrm{FPz}$ at $10 \%$ of the nasion-inion distance.

The horizontal and vertical electro-oculogram movement was recorded by two independent electrodes, placed on the outer canthus of the right eye and below the left eye, respectively. The common reference electrode was placed on the tip of the nose and the ground electrode was located between FPz and Fz. Two more independent electrodes were placed at the mastoids and used later as a re-reference. EEG channels were digitized at a rate of $500 \mathrm{~Hz}$ by an amplifier, and electrode impedance was always kept below $5 \mathrm{k} \Omega$.

\subsection{Data analysis}

To simplify the analysis and improve the signal-to-noise ratio, behavioral and ERP data from stimuli that were symmetrically rotated around $180^{\circ}$ were collapsed separately for normal and mirrored letters. Thus, the rotation angles $50^{\circ}$ and $310^{\circ}, 100^{\circ}$ and $260^{\circ}$, and $150^{\circ}$ and $210^{\circ}$ were considered equivalent (Hamm, Johnson, \& Corballis, 2004; Núñez-Peña \& Aznar-Casanova, 2009).

Median response times for correct responses and percentage of hits were analyzed by ANOVAs, taking Orientation $\left(0^{\circ}, 50^{\circ} / 310^{\circ}, 100^{\circ} / 260^{\circ}\right.$, $150^{\circ} / 210^{\circ}$ ) and Version (Normal, Mirror) as within-subject factors and Group (HMA, LMA) as the between-subjects factor. The $F$ value, the uncorrected degrees of freedom, the probability level following correction, the $\varepsilon$ value (when appropriate), and the $\eta_{p}{ }^{2}$ effect size index for each ANOVA are given. Pairwise comparisons were conducted using t-tests and the Hochberg approach was used to control for the increase in Type I error (Keselman, 1998). Trend analyses were also performed. Finally, whenever the Group effect was significant, an ANCOVA taking spatial anxiety as the covariate was used to control for the effect of this variable. Significance level was set at $p \leq .05$ and only significant results are reported.

The electrophysiological data were pre-processed and analyzed using EEGLAB 14.1.1, a toolbox of MATLAB 9.1.0.441655 (R2016b) software (The MathWorks, Inc). A band-pass filter was set from 0.3 to $30 \mathrm{~Hz}$. Next, an Independent Components Analysis (specifically, the binica algorithm provided by EEGLAB; Delorme \& Makeig, 2004) was used to correct the signal for eye movement-related activity and other artifacts (Mozaffar \& Petr, 2002). Then, epochs for every participant in each experimental condition were averaged, relative to a pre-stimulus baseline consisting of $100 \mathrm{~ms}$ of the preceding electrophysiological activity. Only trials in which the subjects responded correctly were included in the ERP analysis. The mean number of epochs included in each ERP average varied between 17 and 48 for the various types of stimuli used.

After a visual inspection of the ERP waveforms, we decided to capture possible differences between the HMA and LMA groups by determining the mean ERP amplitude for each group in the 450-550 ms window after the stimulus was presented. ERP statistical analysis was performed using data from nine electrodes (F3, Fz, F4, C3, Cz, C4, P3, $\mathrm{PZ}, \mathrm{P} 4)$ and was similar to that described for behavioral responses, except that ANOVA included two more within-subject factors: Frontality (frontal, central and parietal) and Laterality (three levels from left to right).

\section{Results}

\subsection{Behavioral measures}

Response time was significantly affected by Group, $F(1,35)=6.98$, $p=.01, \eta_{p}{ }^{2}=.17$, Orientation, $F(3,105)=242.16, p<.001, \varepsilon=.55$, 
$\eta_{p}^{2}=.87$, and Version, $F(1,35)=76.97, p<.001, \eta_{p}{ }^{2}=.69$. The Orientation $\mathrm{x}$ Version interaction was also significant, $F(3,105)=12.01, p$ $<.001, \varepsilon=.72, \eta_{p}^{2}=.26$. High math-anxious individuals were slower to respond (mean $=704.5 \mathrm{~ms}$, SEM $=29.3 \mathrm{~ms}$ ) than their LMA peers (mean $=596.34 \mathrm{~ms}$, SEM $=28.6 \mathrm{~ms}$ ). Importantly, the ANCOVA showed that the effect of Group on response time remained significant after controlling for the effect of spatial anxiety, $F(1,34)=8.312, p=.007$, $\eta_{p}^{2}=.20$.

Overall, response time increased with angular deviation from upright; all paired comparisons between orientations reached statistical significance (all p-values < .001; means were 537.8, 589.8, 654.9 and $819.2 \mathrm{~ms}$ for $0^{\circ}, 50^{\circ}, 100^{\circ}$ and $150^{\circ}$ respectively; SEMs were $17.1,19.6$, 20.2 and $27.8 \mathrm{~ms}$ respectively). Regarding the effect of Version, in general, participants were faster when responding to normal than to mirrored letters. However, the Versi of Version, in general, participants were faster when responding to normal than to mirrored letters. However, the Version effect varied depending on the orientation, as demonstrated by the Orientation $\mathrm{x}$ Version interaction. Responses to mirrored stimuli were slower than to normal letters at $0^{\circ}(t(36)=10.77$, $p<.001), 50^{\circ}(t(36)=8.92, p<.001)$ and $100^{\circ},(t(36)=6.11, p<$ $.001)$. No differences were found at $150^{\circ}$. Finally, trend analysis showed that the orientation effect was described by a linear and a quadratic trend both for normal $(F(1,35)=362.44, p<.001$ for linear trend and $F(1,35)=113.82, p<.001$ for quadratic trend) and mirrored letters $(F(1,35)=170.90, p<.001$ for linear trend and $F(1,35)=6.47, p=$ .02 for quadratic trend). Fig. 2 shows the RT means and standard errors for normal and mirrored letters in each orientation for both groups.

ANOVA for percentage of hits showed a significant effect of Orientation, $F(3,105)=43.53, p<.001, \varepsilon=.42, \eta_{p}^{2}=.55$, Version, $F(1,35)=11.17, p=.002, \eta_{p}^{2}=.24$, and the Orientation $\mathrm{x}$ Version interaction, $F(3,114)=20.39, p<.001, \varepsilon=.44, \eta_{p}{ }^{2}=.35$. Neither the Group effect nor its interaction with the other factors reached significance. Overall, hits increased with shorter angular disparity from the upright: all paired comparisons between orientations reached statistical significance (all pvalues $\leq .001$ ) except the comparison between $0^{\circ}$ and $50^{\circ}$ (means were 98.1, 98.0, 95.7 and 87.7 for $0^{\circ}, 50^{\circ}, 100^{\circ}$ and $150^{\circ}$ respectively; SEMs were $0.4,0.4,0.6$, and 1.5 respectively). The version effect depended on the orientation, as shown by the Orientation $\mathrm{x}$ Version interaction. Tests of simple effects showed that more hits were made on normal than on mirrored letters at $50^{\circ}(t(36)=2.36$, $p=.024)$, but accuracy was worse for normal than mirrored letters at the larger deviations from upright $(t(36)=2.72, p=.010$ and $t(36)=4.13, p<.001$ for 100 and $150^{\circ}$ respectively). There was no difference in accuracy between the two versions of letters in the upright position. Finally, trend analysis showed that the orientation effect was described by a linear and a quadratic trend both for normal $(F(1,35)=42.7, p<.001$, and $F(1,35)=33.40, p<.001$, for linear and

\section{(A) NORMAL LETTERS}

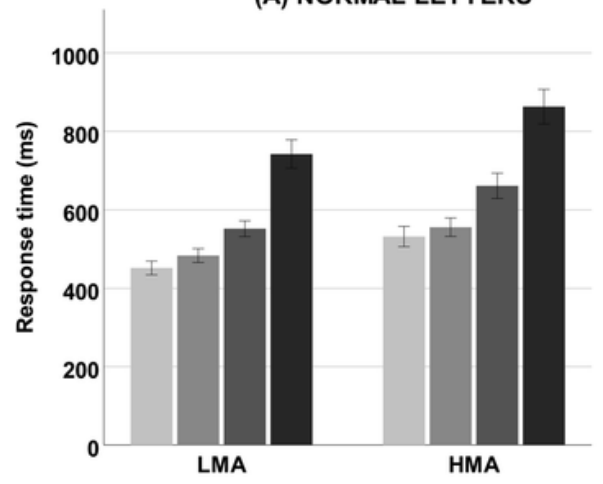

quadratic trend respectively) and mirrored letters $(F(1,35)=6.81, p=$ .013 and $F(1,35)=5.15, p=.03$ respectively).

\subsection{ERP measures}

The overall ANOVA showed significant effects of Orientation, $F(3,105)=16.27, p<.001, \varepsilon=.55, \eta_{p}^{2}=.32$, Orientation $\mathrm{x}$ Version, $F(3,105)=6.15, p=.001, \eta_{p}^{2}=.15$, Orientation x Frontality, $F(6,210)=14.54, p<.001, \varepsilon=.43, \eta_{p}^{2}=.29$, Orientation $\mathrm{x}$ Version $\mathrm{x}$ Frontality, $F(6,210)=6.01, p=.001, \varepsilon=.49, \eta_{p}{ }^{2}=.15$, and Frontality x Laterality x Group, $F(4,140)=4.94, p=.002, \varepsilon=.79, \eta_{p}{ }^{2}=.12$. The main effect of Group was marginally significant, $F(1,35)=3.81, p=$ $.059, \eta_{p}{ }^{2}=.10$, as was the interaction Orientation $\mathrm{x}$ Frontality $\mathrm{x}$ Group $F(6,210)=2.39, p=.084, \varepsilon=.43, \eta_{p}^{2}=.06$.

Núñez-Peña and Aznar-Casanova (2009) reported ERP differences between the processing of normal and mirrored letters in a mental rotation task, and they recommended studying them separately. Since a significant interaction in the present data also showed that the effect of the orientation depended on the version we computed separated ANOVAs for each version. For normal letters, we found significant effects of Orientation, $F(3,105)=18.24, p<.001, \varepsilon=.64, \eta_{p}{ }^{2}=.34$, Orientation $\mathrm{x}$ Frontality, $F(6,210)=17.01, p<.001, \varepsilon=.42, \eta_{p}{ }^{2}=.33$, Orientation x Frontality x Group, $F(6,210)=3.13, p=.038, \varepsilon=.42$, $\eta_{p}{ }^{2}=.08$, and Frontality $\mathrm{x}$ Laterality $\mathrm{x}$ Group, $F(4,140)=3.70, p=$ $.010, \varepsilon=.77, \eta_{p}{ }^{2}=.10$. Separate ANOVAs were performed for each frontality level and the results showed that the Orientation effect was significant at frontal, $F(3,105)=4.43, p=.011, \varepsilon=.78, \eta_{p}{ }^{2}=.11$, central, $F(3,105)=18.84, p<.001, \varepsilon=.64, \eta_{p}^{2}=.35$, and parietal sites, $F(3,105)=26.61, p<.001, \varepsilon=.52, \eta_{p}^{2}=.42$. Trend analysis showed that a linear trend (the more the letter was rotated, the more negative the potential) could be fitted for the three regions: $F(1,35)=6.11, p=$ $.018, \eta_{p}{ }^{2}=.15$ at frontal sites, $F(1,35)=25.66, p<.001, \eta_{p}{ }^{2}=.42$ at central sites, and $F(1,35)=32.92, p<.001, \eta_{p}{ }^{2}=.48$ at parietal sites. The Group effect was significant at parietal sites, $F(1,35)=4.69, p=$ .037, $\eta_{p}{ }^{2}=.12$, the amplitude being more positive for the HMA group.

A more detailed analysis was carried out for normal letters by performing separate ANOVAs for each orientation at the frontal, central and parietal sites. The results showed that amplitude was more positive for the HMA than for the LMA group at $100^{\circ}(F(1,35)=5.66, p=.02$, $\left.\eta_{p}^{2}=.14\right)$, and $150^{\circ}\left(F(1,35)=8.45, p=.006, \eta_{p}^{2}=.19\right)$, only at parietal sites. These differences were maintained after controlling for spatial anxiety in the $100^{\circ}$ condition, $F(1,34)=4.16, p=.049, \eta_{p}^{2}=.11$, and the $150^{\circ}$ condition, $F(1,34)=5.97, p=.02, \eta_{p}{ }^{2}=.14$.

For mirrored letters, we found significant effects of Orientation, $F(3,105)=6.15, p=.001, \varepsilon=.68, \eta_{p}^{2}=.15$, Orientation $x$ Frontality, $F(6,222)=4.85, p=.003, \varepsilon=.50, \eta_{p}^{2}=.12$, and Frontality $\mathrm{x}$ Laterality x Group, $F(4,148)=5.12, p=.002, \varepsilon=.80, \eta_{p}{ }^{2}=.12$. The ef-

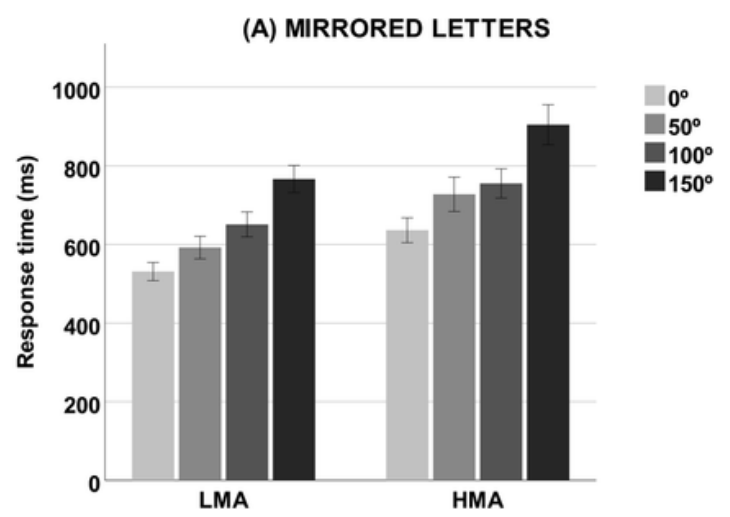

Fig. 2. RT (means and SEM) as a function of stimulus orientation for the LMA and HMA groups for (A) normal and (B) mirrored letters. 
fect of Group was marginally significant, $F(1,35)=3.90, p=.056$, $\eta_{p}{ }^{2}=.10$. Separate ANOVAs were performed for each frontality level and the results showed that the Orientation effect was only significant at central, $F(3,105)=6.28, p=.001, \varepsilon=.73, \eta_{p}{ }^{2}=.15$, and parietal sites, $F(3,105)=8.95, p<.001, \varepsilon=.71, \eta_{p}{ }^{2}=.20$. Trend analyses showed that a linear and a quadratic trend could be fitted at both central $\left(F(1,35)=8.84, p=.005, \eta_{p}^{2}=.20\right.$ and $F(1,35)=4.65, p=.038$, $\eta_{p}{ }^{2}=.12$ for linear and quadratic trend respectively) and parietal sites $\left(F(1,35)=11.65, p=.002, \eta_{p}^{2}=.25\right.$ and $F(1,35)=9.10, p=.005$, $\eta_{p}^{2}=.21$ for linear and quadratic trend respectively).

A more detailed analysis was carried out for mirrored letters by performing separate ANOVAs for each orientation at frontal, central and parietal sites. The results showed that amplitude was more positive for the HMA than for the LMA group at $0^{\circ},(F(1,35)=4.72, p$ $\left.=.037, \eta_{p}{ }^{2}=.12\right), 50^{\circ}\left(F(1,35)=5.08, p=.031, \eta_{p}{ }^{2}=.13\right), 100^{\circ}$ $\left(F(1,35)=5.54, p=.02, \eta_{p}^{2}=.14\right)$, and $150^{\circ}(F(1,35)=8.65, p=$ $.006, \eta_{p}{ }^{2}=.20$ ), only at parietal sites. These differences were maintained after controlling for spatial anxiety in the $50(F(1,34)=5.07, p=$ $\left..031, \eta_{p}{ }^{2}=.13\right)$, the $100^{\circ}\left(F(1,34)=5.05, p=.031, \eta_{p}{ }^{2}=.13\right)$, and the $150^{\circ}$ condition $\left(F(1,34)=8.35, p=.007, \eta_{p}^{2}=.20\right)$. They were marginally significant at $0^{\circ}, F(1,34)=2.96, p=.095, \eta_{p}^{2}=.08$.

Finally, although (as stated above) the interaction Group $\mathrm{x}$ Version was not significant in the initial examination, we performed a further analysis focused on the parietal sites, given that the P3b amplitude is larger in these areas. An ANOVA taking Orientation, Version and Laterality as within-subject factors and Group as the between-subjects factor was conducted. Results showed significant effects for Orientation, $F(3,105)=23.38, p<.001, \varepsilon=.52, \eta_{p}^{2}=.39$, Version, $F(1,35)=5.60$, $p=.024, \eta_{p}^{2}=.14$, Version $\mathrm{x}$ Orientation, $F(3,105)=9.52, p<.001$, $\eta_{p}^{2}=.21$, and Group, $F(1,35)=5.75, p=.022, \eta_{p}^{2}=.14$. Importantly, these results replicated those by Núñez-Peña and Aznar-Casanova (2009), showing a version effect that depended on the orientation. Simple effects analyses showed that amplitude was more negative for mirrored than for normal letters at the upright and the $50^{\circ}$ positions $\left(F(1,35)=6.41, p=.016, \eta_{p}{ }^{2}=.15\right.$ and $F(1,35)=23.58, p<.001$, $\eta_{p}{ }^{2}=.40$, respectively). Once again, HMA showed larger amplitudes than their LMA peers. Fig. 3 shows the ERP grand-averages at Pz for normal and mirrored letters in each orientation for the LMA and HMA groups and Fig. 4 shows the scalp topography for normal and mirrored letters in each orientation for the LMA and HMA groups in the $450-550 \mathrm{~ms}$ window.

\section{Discussion}

In the experiment reported here, we examined differences between high and low math-anxious individuals in a mental rotation task by recording behavioral measures (i.e., RT and hit rate) and brain activity (i.e., ERPs). Previous studies (e.g., Ferguson et al., 2015; Maloney et al., 2012) have shown that math-anxious individuals are less skilled in small-scale spatial processing than their non-math-anxious peers. Moreover, other studies (Suárez-Pellicioni et al., 2013a, 2014) have reported that HMA individuals might have an attentional control deficit when they perform numerical tasks and that they might need to invest more attentional resources than their LMA counterparts. The present study was designed to examine whether HMA individuals might also need to invest a greater amount of attentional resources in spatial tasks (e.g., a mental rotation task) than their LMA peers.

Regarding behavioral measures, the well-known mental rotation effect (Shepard \& Metzler, 1971) was replicated: response time increased with larger deviations from upright both for the HMA and LMA groups. Interestingly, individuals high in math anxiety were slower than their LMA peers, but the groups did not differ in accuracy. PET and ACT (Eysenck \& Calvo, 1992; Eysenck et al., 2007) distinguish between performance effectiveness (i.e., the quality of task performance) and processing efficiency (i.e., the effort or resources invested to achieve a given effectiveness level); our group differences in RT suggest that HMA individuals might need to invest more processing effort in order to achieve the same level of accuracy as their non-math-anxious counterparts.

In terms of the ERPs, the orientation effect (Heil, 2002; Núñez-Peña \& Aznar-Casanova, 2009; Núñez-Peña et al., 2005) was also replicated in both groups. We observed increasing negativity with increasing angular displacement from upright (i.e., the RRN). Moreover, our results also replicated those of Núñez-Peña and Aznar-Casanova (2009), showing a version effect that depended on the orientation: the amplitude was more negative for mirrored than for normal letters in the $0^{\circ}$ and $50^{\circ}$ orientations at parietal sites. Núñez-Peña and Aznar-Casanova suggested that this might reflect a rotation out of the plane involved in the processing of mirror-rotated letters.

As for ERP differences between HMA and LMA individuals, interestingly, amplitude was more positive for the HMA than for the LMA group in the $100^{\circ}$ and $150^{\circ}$ condition for normal letters and in the 0 , 50, 100 and $150^{\circ}$ condition for mirrored letters. Because it has been suggested that the RRN is superimposed by a P3b, the latter being a

\section{LOW MATH ANXIETY}

\section{HIGH MATH ANКIETY}
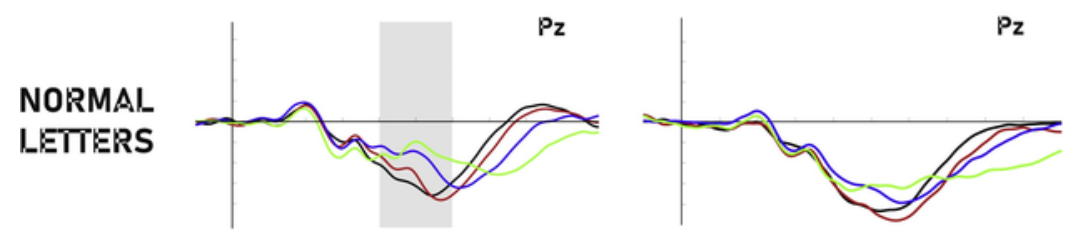

Pz

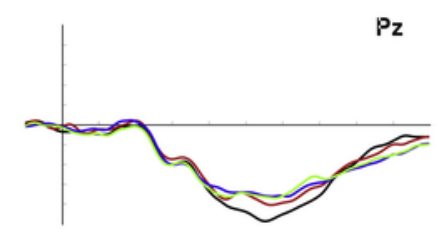

MIRROR LETTERS
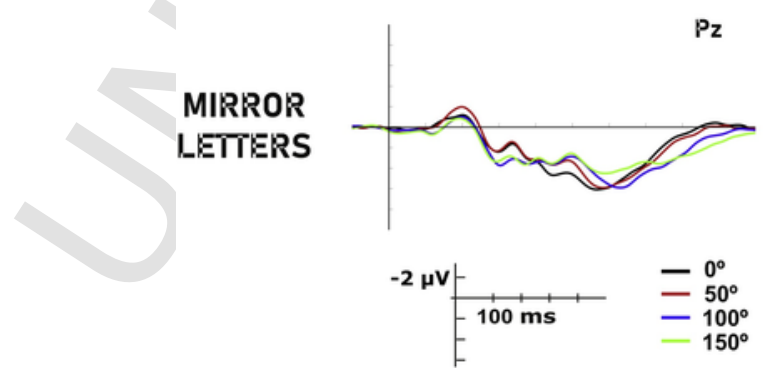

Fig. 3. Grand-average ERPs at Pz for normal and mirrored letters in each orientation for the LMA and HMA groups. The time interval for the RRN is shaded. 


\section{(A) NORMAL LETTERS}

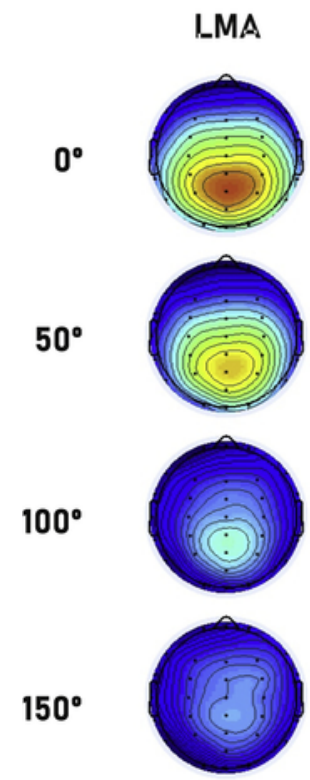

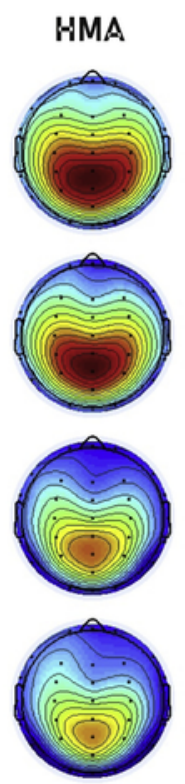

(B) MIRRORED LLETTERS

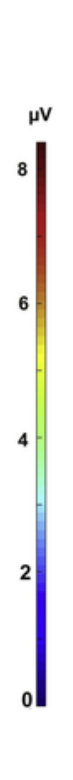

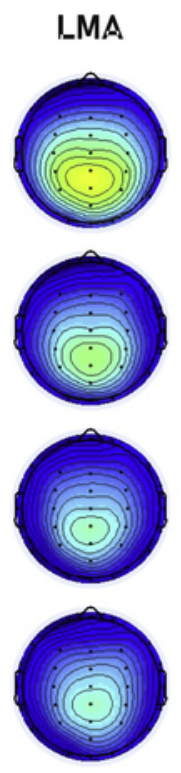

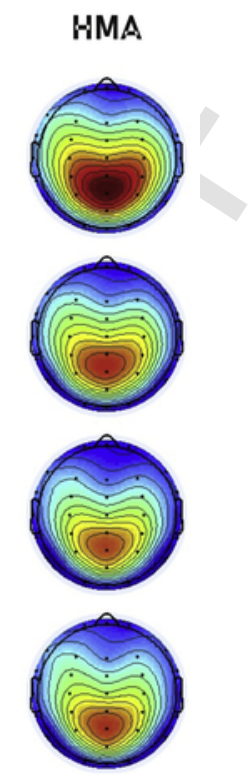

Fig. 4. Scalp topography in each orientation for the LMA and HMA groups in the 450-550 ms window for (A) normal and (B) mirrored letters.

marker of the amount of attentional resources invested in performing the given task (Kok, 2001, 1997; Polich, 2007), this larger positivity for HMA individuals confirms that they might need to invest more attentional resources than their LMA peers to perform the mental rotation task.

However, why were P3b group differences only found in some of the types of stimulus (specifically, in larger deviations from upright in normal letters and in all mirrored letters)? Firstly, it has been widely demonstrated that mental rotation is not needed for small deviations from upright (e.g., $50^{\circ}$ ) in over-learned familiar stimuli like the normal letters used in the present study. This has been found both for behavioral measures (Cooper \& Shepard, 1975; Koriat \& Norman, 1985) and ERPs (Núñez-Peña \& Aznar-Casanova, 2009). This would explain why no group differences were found in the $50^{\circ}$ normal letter condition; mental rotation would not be used to perform the task in this type of stimulus. However, unfamiliar stimuli, like the mirrored letters presented here, would need to be rotated even for small deviations from upright (i.e., $50^{\circ}$ ). Secondly, it has been suggested that for decisions on mirrored letters the stimulus needs to be mentally rotated first in the picture plane, to place it in the vertical upright position, and then out of the plane, to place it in the normal upright position (Hamm et al., 2004; Núñez-Peña \& Aznar-Casanova, 2009). Therefore, mental rotation would be needed for all mirrored stimulus, even for the upright mirrored letters. All in all, the larger P3b amplitude found in the present study for highly math-anxious individuals only when dealing with larger deviations in normal letters and all deviated mirrored letters suggests that they may only need to invest greater attentional effort when the task becomes more difficult and requires more cognitive resources.

Although the dominant view regarding P3b links it to stimulus-related rather than to response-related processing (i.e., organizing the response), some authors have suggested that it might be related equally to both stimulus and response (e.g., Falkenstein, Hohnsbein, \& Hoormann, 1994; Verleger, Jaśkowski, \& Wascher, 2005). Verleger et al. (2005) suggested that P3b reflects a function that bridges perceptual (stimulus) processing with action (response) processing. In particular, they linked P3b to the process of monitoring whether the decision to classify the stimulus and the subsequent action has led to appropriate processing. According to this proposal, the larger P3b amplitudes found for HMA individuals in the present study might suggest that these individuals might need to use more cognitive resources to evaluate or regulate their decision-making in mental rotation tasks. Unfortunately the present results do not allow us to discriminate between these two interpretations, and further research is needed to better understand the cognitive process reflected by P3b in the context of mental rotation tasks.

Three relevant variables related to mathematical anxiety were controlled in the current study. The first variable was trait anxiety. Individuals high in math anxiety also report high levels of trait anxiety (Hembree, 1990) and, according to the ACT (Eysenck et al., 2007), trait anxiety impairs processing efficiency. To rule out the possibility that our group differences were due to trait anxiety, our groups were formed in such a way that they differed in math anxiety but not in trait anxiety. Second, spatial anxiety was also controlled. Spatial anxiety is positively related to mathematical anxiety and negatively related to performance in mental rotation tasks (Lawton, 1994). In our study, although HMA individuals had more spatial anxiety than their LMA peers, when this latter variable was introduced as a covariate in the analysis, the group effect was maintained. Thus, we were able to rule out the possibility that our group differences were due to spatial anxiety. Finally, gender is associated with math anxiety and spatial ability (e.g., Sokolowski et al., 2019), so this variable was controlled in the experiment by selecting only women as participants. We acknowledge this as a limitation of our study, since it is not clear that its conclusions apply also to highly math-anxious men.

Another possible limitation of this study is the fact that the $0^{\circ}$ condition was presented twice as often as the other conditions. On the one hand, given that deviated clockwise and counterclockwise orientations are collapsed in the analyses, doubling the number of trials in the $0^{\circ}$ position provides us with the same number of trials for each orientation. As such, it is a standard procedure in ERP studies with mental rotation tasks (e.g., Lust, Geuze, Wijers, \& Wilson, 2006; Núñez-Peña \& Aznar-Casanova, 2009). On the other hand, P3 amplitude has been related to the subjective probability of the stimulus (Polich, 2007) and therefore, one might wonder whether the different proportions of stimuli might have affected our results. However, it is worth bearing in mind that this lower frequency was identical across all the deviated 
stimuli, and yet we found a greater rotation-related negativity as deviation increased. More importantly, frequencies of presentation were the same for all participants, and so this explanation does not invalidate our conclusions concerning group differences.

To conclude, we would like to suggest a tentative explanation for the extra resources required by HMA individuals in spatial processing tasks. Previous studies, based on the ACT (Eysenck et al., 2007), suggested that HMA individuals could have low achievement in math-related tasks because their worries and low self-confidence would distract them from performing well in the task at hand (Suárez-Pellicioni et al., 2013a, 2014). Our study might extend this explanation to the association between math anxiety and spatial processing. Ferguson et al. (2015) found that HMA individuals self-reported lower spatial ability and more spatial anxiety than their LMA peers. As in the case of numerical tasks, HMA individuals' lower self-confidence in their spatial ability could give rise to ruminations about their low capacity when they perform this type of task. These ruminations might consume the attentional resources of the central executive system required to perform the spatial task, and then HMA individuals would need to invest more resources in order to perform the task; they would then be slower and show a larger P3b than their LMA counterparts. Such a conclusion fits nicely both with our results and with those of Ferguson et al. (2015). However, we acknowledge two limitations of our study, both of which are related to the heterogeneity of spatial abilities (Hegarty, Montello, Richardson, Ishikawa, \& Lovelace, 2006). First, we did not measure self-reported spatial skills; although Ferguson et al. did measure them, they only assessed the perceived sense of direction, i.e., a large-scale ability. Second, the Spatial Anxiety Scale (Lawton, 1994), used by ourselves and by Ferguson et al. (2015), measures anxiety when having to use large-scale abilities. Ferguson et al. (2015) found that SAS scores significantly correlated with both small- and large-scale abilities. However, given that our experimental task involved small-scale skills (rotation), and that only small-scale spatial abilities seem to predict math anxiety, future studies might want to use a spatial anxiety test that (also) assesses anxiety when facing small-scale situations. In this context, a questionnaire recently developed by Lyons et al. (2018) might be useful, since it measures spatial anxiety in navigation, mental manipulation and imagery.

To sum up, the results of this study extend those of Suárez-Pellicioni et al. (2013a), Suárez-Pellicioni, Núñez-Peña, and Colomé (2013, 2014) on math-related tasks to spatial processing tasks, suggesting that math-anxious individuals may need to invest more attention and cognitive resources than their low math-anxious counterparts in mental rotation tasks. This greater investment of attentional resources was demonstrated in both the behavioral and brain responses and may be one of the factors underlying the low small-scale spatial ability in highly math-anxious individuals.

\section{Funding}

This research was supported by the Spanish Ministry of Economy and Competitiveness and the European Regional Development Fund, FEDER, under grant PSI2015-69915-R (MINECO/FEDER), and by the Generalitat de Catalunya under grants SGR2017-974, SGR2017-48 and 2018FI-B-00346.

\section{Uncited references} (2015)

\section{Acknowledgments}

We thank Francisco Javier Diaz Santaella for developing the E-prime program for task presentation and Martina Frontini Mensa and Sara Muntadas Castelló for their help with data collection.

\section{References}

Alexander, L., Martray, C., 1989. The development of an abbreviated version of the Math ematics Anxiety Rating Scale. Measurement and Evaluation in Counseling and Development22, 143-150.

Ashcraft, M.H., Ridley, K., 2005. Math anxiety and its cognitive consequences: A tutorial review. In: Campbell, J.I.D. (Ed.), Handbook of mathematical cognition. Psychology, New York, pp. 315-325.

Barceló, F., Periáñez, J.A., Nyhus, E., 2008. An information theoretical approach to task-switching: Evidence from cognitive brain potentials in humans. Frontiers in $\mathrm{Hu}$ man Neuroscience 1, 13.

Cooper, L.A., Shepard, R.N., 1973. Chronometric studies of the rotation of mental images. In: Chase, W.G. (Ed.), Visual information processing. Academic Press, New York.

Cooper, L.A., Shepard, R.N., 1975. Mental transformation in the identification of left and right hands. Journal of Experimental Psychology Human Perception and Performance 1 (1), 48-56.

de Hevia, M.D., Vallar, G., Girelli, L., 2008. Visualizing numbers in the mind's eye: The role of visuo-spatial processes in numerical abilities. Neuroscience and Biobehavioral Reviews 32 (8), 1361-1372.

Dehaene, S., 1992. Varieties of numerical abilities. Cognition 44, 1-42.

Delorme, A., Makeig, S., 2004. EEGLAB: An open source toolbox for analysis of single-trial EEG dynamics including independent component analysis. Journal of Neuroscience Methods 134, 9-21.

Duncan-Johnson, C.C., Donchin, E., 1977. On quantifying surprise: The variation of event-related potentials with subjective probability. Psychophysiology 14, 456-467.

Eysenck, M.W., Calvo, M.G., 1992. Anxiety and performance: The processing efficiency theory. Cognition \& Emotion 6 (6), 409-434.

Eysenck, M.W., Derakshan, N., Santos, R., Calvo, M.G., 2007. Anxiety and cognitive performance: Attentional control theory. Emotion 7 (2), 336-353.

Falkenstein, M, Hohnsbein, J., Hoormann, J., 1994. Time pressure effects on late components on late components of the event-related potential. Journal of Psychophysiology 8, 22-30.

Ferguson, A.M., Maloney, E.A., Fugelsang, J., Risko, E.F., 2015. On the relation between math and spatial ability: The case of math anxiety. Learning and Individual Differences 39, 1-12

Fias, W., van Dijck, J., Gevers, W., 2011. How is number associated with space? The role of working memory. In: Dehaene, E., Brannon, S. (Eds.), Space, tme and number in the brain. Academic Press, London (UK), pp. 249-265.

Gootjes, L., Bruggeling, E.C., Magnée, T., Van Strien, J.W., 2008. Sex differences in the latency of the late event-related potential mental rotation effect. Neuroreport 19 (3), 349-353.

Gray, H.M., Ambady, N., Lowenthal, W.T., Deldin, P., 2004. P300 as an index of attention to self-relevant stimuli. Journal of Experimental Social Psychology 40, 216-224.

Hamm, J.P., Johnson, B.W., Corballis, M.C., 2004. One good turn deserves another: An event-related brain potential study of rotated mirror-normal letter discriminations. Neuropsychologia 42 (6), 810-820.

Hegarty, M., Montello, D.R., Richardson, A.E., Ishikawa, T., Lovelace, K., 2006. Spatial abilities at different scales: Individual differences in aptitude-test performance and spatial-layout learning. Intelligence 34 (2), 151-176.

Hegarty, M., Richardson, A.E., Montello, D.R., Lovelace, K., Subbiah, I., 2002. Development of a self-report measure of environmental spatial ability. Intelligence 30 (5), 425-447.

Heil, M., 2002. The functional significance of ERP effects during mental rotation. Psychophysiology 39, 535-545.

Heil, M., Rolke, B., 2002. Towards a chronopsychophysiology of mental rotation. Psychophysiology 39, 414-422.

Hembree, R., 1990. The nature, effects, and relief of mathematics anxiety. Journal for Research in Mathematics Education 21 (1), 33-46.

Hubbard, E.M., Piazza, M., Pinel, P., Dehaene, S., 2005. Interactions between number and space in parietal cortex. Nature Reviews Neuroscience 6 (6), 435-448.

Johnson Jr., R., 1988. The amplitude of the P300 component of the event-related potential: Review and synthesis. In: In: Ackles, P.K., Jennings, J.R., Coles, M.G.H. (Eds.), Advances in psychophysiology Vol. 3, JAI Press, Inc., Greenwich, CT, pp. 69-137.

Keselman, H.J., 1998. Testing treatment effects in repeated measures designs: An update for psychophysiological researchers. Psychophysiology 35 (4), 470-478.

Kok, A., 1997. Event-related-potential (ERP) reflections of mental resources: A review and synthesis. Biological Psychology 45 (1-3), 19-56.

Kok, A., 2001. On the utility of P300 amplitude as a measure of processing capacity. Psychophysiology 38, 557-577.

Koriat, A., Norman, J., 1985. Reading rotated words. Journal of Experimental Psychology Human Perception and Performance 11 (4), 490-508. 
Lawton, C.A., 1994. Gender differences in way-finding strategies: Relationship to spatial ability and spatial anxiety. Sex Roles 30 (11), 765-779.

Levine, S.C., Foley, A., Lourenco, S., Ehrlich, S., Ratliff, K., 2016. Sex differences in spatial cognition: Advancing the conversation. Wiley Interdisciplinary Reviews Cognitive Science 7 (2), 127-155.

Lippa, R.A., Collaer, M.L., Peters, M., 2010. Sex differences in mental rotation and line angle judgments are positively associated with gender equality and economic development across 53 nations. Archives of Sexual Behavior 39 (4), 990-997.

Lohman, D.F., 1994. Spatial ability. In: In: Sternberg, R.J. (Ed.), Encyclopedia of intelligence Vol. 2, Macmillan, New York, pp. 1000-1007.

Lubinski, D., 2010. Spatial ability and STEM: A sleeping giant for talent identification and development. Personality and Individual Differences 49 (4), 344-351.

Lust, J.M., Geuze, R.H., Wijers, A.A., Wilson, P.H., 2006. An EEG study of mental rotation-related negativity in children with developmental coordination disorder. Health and Development 32 (6), 649-663.

Lyons, I.M., Ramirez, G., Maloney, E.A., Rendina, D.N., Levine, S.C., Beilock, S.L., 2018. Spatial Anxiety: A novel questionnaire with subscales for measuring three aspects of spatial anxiety. Journal of Numerical Cognition 4 (3), 526-553.

Maloney, E.A., Waechter, S., Risko, E.F., Fugelsang, J.A., 2012. Reducing the sex difference in math anxiety: The role of spatial processing ability. Learning and Individual Differences 22 (3), 380-384.

Mikhailova, E.S., Slavutskaya, A.V, Gerasimenko, N.Y., 2012. Gender differences in the recognition of spatially transformed figures: Behavioral data and event-related potentials (ERPs). Neuroscience Letters 524 (2), 74-78.

Mix, K.S., Cheng, Y.L., 2012. The relation between space and math: Developmental and educational implications. Advances in Child Development and Behavior 42, 197-243.

Mozaffar, S., Petr, D., 2002. Artifact extraction from EEG data using independent component analysis. Kansas: U. S. The University of Kansas Center for Research, Inc.. Information Telecommunication and Technology Center, (Tech. Rep. No. ITTC-FY2003-TR-03050-02)

Núñez-Peña, M.I., Aznar-Casanova, J.A., 2009. Mental rotation of mirrored letters: Evidence from event-related brain potentials. Brain and Cognition 69 (1), 180-187.

Núñez-Peña, M.I., Aznar-Casanova, J.A., Linares, D., Corral, M.J., Escera, C., 2005. Effects of dynamic rotation on event-related brain potentials. Brain Research Cognitive Brain Research 24 (2), 307-316.

Núñez-Peña, M.I., Suárez-Pellicioni, M., 2014. Less precise representation of numerical magnitude in high math-anxious individuals: An ERP study of the size and distance effects. Biological Psychology 103, 176-183.

Núñez-Peña, M.I., Suárez-Pellicioni, M., Guilera, G., Mercadé-Carranza, C., 2013. A Spanish version of the short mathematics anxiety rating scale (sMARS). Learning and Individual Differences 24, 204-210.

Peronnet, F., Farah, M.J., 1989. Mental rotation: An event-related potential study with a validated mental rotation task. Brain and Cognition 9, 279-288.

Pinel, P., Piazza, M., Le Bihan, D., Dehaene, S., 2004. Distributed and overlapping cerebral representations of number, size, and luminance during comparative judgments. Neuron 41 (6), 983-993.

Polich, J., 2007. Updating P300: An integrative theory of P3a and P3b. Clinical Neurophysiology 118 (10), 2128-2148.
Reilly, D., Neumann, D.L., 2013. Gender-role differences in spatial ability: A meta-analytic review. Sex Roles 68 (9-10), 521-535.

Richardson, F.C., Suinn, R.M., 1972. The mathematics anxiety rating scale: Psychometric data. Journal of Counceling Psychology 19 (6), 551-554.

Rösler, F., Schumacher, G., Sojka, B., 1990. What the brain reveals when it thinks. Event-related potentials during mental rotation and mental arithmetic. The German Journal of Psychology 14, 185-203.

Shepard, R.N., Metzler, J., 1971. Mental rotation of three-dimensional objects. Science 171 (3972), 701-703.

Sokolowski, H.M., Hawes, Z., Lyons, I.M., 2019. What explains sex differences in math anxiety? A closer look at the role of spatial processing. Cognition 182, 193-212.

Spielberger, C.D., Gorsuch, R., Lushene, R., 2008. Cuestionario de ansiedad Estado-Rasgo, STAI. TEA Ediciones, Madrid.

Spielberger, C.D., Gorsuch, R., Lushene, R., Vagg, P.R., Jacobs, G.A., 1983. Manual for the State-Trait anxiety inventory. Consulting Psychology Press, Palo Alto, CA.

Suárez-Pellicioni, M., Núñez-Peña, M.I., Colomé, A., 2013. Mathematical anxiety effects on simple arithmetic processing efficiency: An event-related potential study. Biological Psychology 94 (3), 517-526.

Suárez-Pellicioni, M., Núñez-Peña, M.I., Colomé, , 2013. Abnormal error monitoring in math-anxious individuals: Evidence from error-related brain potentials. PloS One 8 (11), e81143.

Suárez-Pellicioni, M., Núñez-Peña, M.I., Colomé, , 2014. Reactive recruitment of attentional control in math anxiety: An ERP study of numeric conflict monitoring and adaptation. PLoS One 9 (6), e99579.

Suárez-Pellicioni, M., Núñez-Peña, M.I., Colomé, , 2015. Attentional bias in high math-anxious individuals: Evidence from an emotional Stroop task. Frontiers in Psychology 6, 1577.

Suárez-Pellicioni, M., Núñez-Peña, M.I., Colomé, , 2016. Math anxiety: A review of its co nitive consequences, psychophysiological correlates, and brain bases. Cognitive, Affective \& Behavioural Neuroscience 16 (1), 3-22.

Thayer, Z.C., Johnson, B.W., 2006. Cerebral processes during visuo-motor imagery of hands. Psychophysiology 43, 401-412.

Verleger, R., Jaśkowski, P., Wascher, E., 2005. Evidence for an integrative role of P3b in linking reaction to perception. Journal of Psychophysiology 19 (3), 165-181.

Voyer, D., Voyer, S.S., Bryden, M.P., 1995. Magnitude of sex differences in spatial abilities: A meta-analysis and consideration of critical variables. Psychological Bulletin 117 (2), $250-270$.

Wai, J., Lubinski, D., Benbow, C.P., 2009. Spatial ability for STEM domains: Aligning over 50 years of cumulative psychological knowledge solidifies its importance. Journal of Educational Psychology 101 (4), 817-835.

Wang, L., Cohen, A.S., Carr, M., 2014. Spatial ability at two scales of representation: A meta-analysis. Learning and Individual Differences 36, 140-144.

Wijers, A.A., Otten, L.J., Feenstra, S., Mulder, G., Mulder, L.J.M., 1989. Brain potentials during selective attention, memory search, and mental rotation. Psychophysiology 26 , $452-467$. 